\title{
Expression of granulocyte colony-stimulating factor 3 receptor in the spinal dorsal horn following spinal nerve ligation-induced neuropathic pain
}

\author{
ENJI ZHANG ${ }^{1,2^{*}}$, SUNYEUL LEE $^{3 *}$, MIN-HEE YI ${ }^{1,4}$, YONGSHAN NAN ${ }^{2}$, YINSHI XU $^{2}$, \\ NARA SHIN ${ }^{1,3}$, YOUNGKWON KO ${ }^{3}$, YOUNG HO LEE ${ }^{1}$, WONHYUNG LEE ${ }^{3}$ and DONG WOON KIM ${ }^{1}$
}

\begin{abstract}
${ }^{1}$ Department of Anatomy and Medical Science, Brain Research Institute, Chungnam National University School of Medicine, Daejeon 301-747, Republic of Korea; ${ }^{2}$ Department of Anesthesiology, Yanbian University Hospital, Yanbian, Jilin 133000, P.R. China; ${ }^{3}$ Department of Anesthesia and Pain Medicine, Chungnam National University Hospital, Daejeon 301-747, Republic of Korea; ${ }^{4}$ Department of Neuroscience and Cell Biology,

The University of Texas Medical Branch School of Medicine, Galveston, TX 77555, USA
\end{abstract}

Received April 13, 2016; Accepted May 11, 2017

DOI: $10.3892 / \mathrm{mmr} .2017 .6853$

\begin{abstract}
In previous studies that have profiled gene expression in patients with complex regional pain syndrome (CRPS), the expression of granulocyte colony-stimulating factor 3 receptor (G-CSFR) was elevated, as were a number of pain-associated genes. The present study determined the expression of G-CSFR and the mechanisms by which it may affect hypersensitivity, focusing on the signal transducer and activator of transcription 3 (STAT3)/transient receptor potential cation channel subfamily V 1 (TRPV1) signaling pathway in particular, which is an important mediator of pain. Following L5 spinal nerve ligation (SNL) surgery, the protein and mRNA levels of G-CSFR increased in the ipsilateral spinal dorsal horn when compared with the sham and/or contralateral control. Double immunofluorescence further demonstrated that G-CSFR colocalized with TRPV1 and phosphorylated STAT in the neurons of the spinal dorsal horn. G-CSF treatment led to an increase in G-CSFR and TRPV1 expression and phosphorylation of STAT3. These results indicate that
\end{abstract}

Correspondence to: Dr Dong Woon Kim, Department of Anatomy and Medical Science, Brain Research Institute, Chungnam National University School of Medicine, 266 Munhwa Road, Chung, Daejeon 301-747, Republic of Korea

E-mail: visnu528@cnu.ac.kr

Dr Wonhyung Lee, Department of Anesthesia and Pain Medicine, Chungnam National University Hospital, 282 Munhwa Road, Daejeon 301-747, Republic of Korea

E-mail:whlee@cnu.ac.kr

*Contributed equally

Key words: granulocyte colony-stimulating factor 3, granulocyte colony-stimulating factor 3 receptor, transient receptor potential cation channel subfamily V 1, spinal cord, neuropathic pain
G-CSF-induced G-CSFR expression may activate TRPV1 by promoting phosphorylation of STAT3. Collectively, the results suggest, for the first time, that the expression of G-CSFR in neurons following peripheral nerve injury may be involved in the induction and maintenance of neuropathic pain through the STAT3 and TRPV1 signaling pathway.

\section{Introduction}

The granulocyte colony-stimulating factor receptor (G-CSFR), also known as Cluster of Differentiation 114 (CD114), is encoded by the CSF3R gene in humans. G-CSFR is a cell-surface receptor for the granulocyte colony-stimulating factor (G-CSF). G-CSF is encoded on chromosome 17 (q11-22) and thus differs from other growth factors including granulocyte macrophage (GM)-CSF, interleukin (IL)-3, IL-4 and IL-5 which are encoded on chromosome 5 (1). $\mathrm{G}-\mathrm{CSF}$ is an important cytokine for the regulation of the maturation, proliferation and differentiation of neurotrophic granulocyte precursor cells and is used to treat patients with granulopenia $(2,3)$. These responses are initiated by interactions between G-CSF and the G-CSFR, which is expressed on neutrophils, neutrophil precursors and some leukemic cell lines. G-CSF binding triggers receptor activation and a variety of signaling cascades, including the Janus kinase (JAK)-signal transducer and activator of transcription (STAT) and mitogen-activated protein kinase (MAPK) signaling pathways $(4,5)$. As the name suggests, G-CSFR is found in a number of myeloproliferative tissue and cell types, including macrophages, natural killer cells, T cells and platelets (6). However, the human fetus expresses G-CSF receptors in the majority of tissue types, particularly in renal and gastrointestinal tract tissues, hemopoietic and neural stem cells, and in various areas of the brain, particularly the radial glia, during the development of the central nervous system (7). In adult humans, G-CSFR is also expressed on endothelial cells and fibroblasts in the liver (also previously observed in rats and mice), the prostate, brain and neurons $(8,9)$. 
Our previous study demonstrated that the gene expression of G-CSFR was significantly increased in patients with complex regional pain syndrome (CRPS) (10). In addition, G-CSFR was functionally expressed in sensory nerves (11). It was also revealed that sensory nerve-specific knockdown of G-CSFR attenuated tumor-associated pain. Therefore, G-CSF may be important in tumor-nerve interactions and G-CSFR on primary afferent nerve fibers may be potential therapeutic targets for cancer-associated pain. Due to these previous works, the focus of the present study was the dorsal horn as a site of pain sensory sensitization, including one for neuropathic pain. Therefore, the role of G-CSFR in the dorsal horn of the spinal cord was investigated in an animal model of neuropathic pain. Nerve lesions in neuropathic pain trigger molecular alterations in nociceptive neurons, which become abnormally sensitive and develop pathological spontaneous activity such as upregulation of sodium channels and receptors, including transient receptor potential cation channel subfamily V 1 (TRPV1) receptors, menthol-sensitive transient receptor potential cation channel subfamily $\mathrm{M}$ member 8 (TRPM8) receptors and $\alpha$-receptors (12). G-CSF signalling in nociceptors has previously been associated with hyperalgesia to thermal and mechanical stimuli (11). The modulation of TRPV1 expression by G-CSF signaling in sensory neurons is particularly notable as TRPV1 is an important mediator for the development of pain. Thus, the present study investigated whether G-CSFR expression in the spinal nerve ligation (SNL)-induced dorsal horn was associated with abnormally sensitive and spontaneously active nociceptive neurons, particularly that associated with TRPV1 signalling.

\section{Materials and methods}

Animals and surgical procedures. A total of 64 male Sprague-Dawley rats (aged 6-8 weeks; 180-200 g) were purchased from Koatech, Inc. (Pyeongtaek. Republic of Korea). The rats were housed in cages at a temperature of $22-25^{\circ} \mathrm{C}$, humidity of $40-60 \%$ and a standard $12 \mathrm{~h}$ light/dark cycle with free access to water and food. All experiments were carried out with the approval of the Animal Care and Use Committee at the Chungnam National University (approval no: CNUH-014-P0005) and were consistent with the ethical guidelines of the National Institutes of Health (Bethesda, MD, USA) and the International Association for the Study of Pain (Seattle, WA, USA). The present study applied the SNL animal model developed by Kim and Chung (13). Briefly, under anesthesia, the left lumber (L) 6 was carefully removed to visually identify the L4 and L5 spinal nerves. The left L5 spinal nerve was tightly ligated with a 3-0 silk thread. The surgical procedure for the sham group $(n=21)$ was identical to that of the SNL group ( $n=21)$, except the spinal nerves were not ligated. Naïve groups that did not receive surgery were also used $(n=21)$.

Von Frey withdrawal threshold. Mechanical paw withdrawal thresholds (PWTs) were measured using the up-down testing paradigm 1 day prior to surgery and on days 3, 7, 10 and 14 following SNL or sham surgery. Von Frey hairs (Touch Test $^{\mathrm{TM}}$ Sensory Evaluator kit of 20; cat. no. 39337500; Leica Biosystems Nussloch GmbH, Nussloch, Germany) in log increments of force $(0.007,0.016,0.4,0.6,1,1.4,2,4,6,8,10$ and $15 \mathrm{~g}$ ) were applied for a duration of 4-6 s to the region between the foot pads in the plantar region of the hind paw. The $2 \mathrm{~g}$ stimulus was applied first. If a positive response was observed, the next smaller Von Frey hair was used; if a negative response was observed, the next higher force was applied.

CatWalk gait analysis. The animals traversed a walkway with a glass floor located in a darkened room. In general, rats are able to cross the CatWalk runway easily and at a constant speed. CatWalk gait analysis was performed at days 0, 5, 10, and 14 of the experiment. The CatWalk analysis system consists of a glass walkway with a white fluorescent source, which produces light rays that are reflected internally. When an object touches the glass runway, the light is reflected downwards, where it is detected by a video camera. This signal is then digitized and analyzed by the CatWalk program software (CatWalk XT version 10.5.505; Noldus Information Technology Inc., Lessburg, VA, USA).

Immunohistochemistry and double immunofluorescence. Immunohistochemistry was performed 14 days following surgery. Rats were anesthetized with sodium pentobarbital (50 mg/kg intraperitoneally) and perfused transcardially with heparinized phosphate-buffered saline (PBS; pH 7.4), followed by perfusion with $4 \%$ paraformaldehyde for $15 \mathrm{~min}$. Enlarged lumbar (L4-L6) regions of the spinal cords were immediately removed, immersed in the same fixative overnight at $4^{\circ} \mathrm{C}$, and then embedded in paraffin. Sections $(4 \mu \mathrm{m})$ of the paraffin-embedded tissue were deparaffinized and rehydrated in a graded alcohol series. The antigen was retrieved in $0.01 \mathrm{M}$ citrate buffer $(\mathrm{pH}$ 6.0) by heating samples in a microwave vacuum histoprocessor (RHS-1; Milestone SRL, Sorisole, BG, Italy) at a controlled final temperature of $121^{\circ} \mathrm{C}$ for $15 \mathrm{~min}$. For immunohistochemical analyses, endogenous peroxidase activity was blocked using $0.3 \%$ hydrogen peroxide at room temperature. The sections were treated with Protein Block solution (Dako; Agilent Technologies, Inc., Santa Clara, CA, USA) at room temperature for $20 \mathrm{~min}$ and then incubated with specific polyclonal antisera against anti-G-CSFR (dilution, 1:200; cat. no. bs-2574R; BIOSS, Beijing, China) overnight in a humidity chamber at $4^{\circ} \mathrm{C}$. Following washing with PBS, the tissues were exposed to biotinylated goat anti-rabbit immunoglobulin G (IgG) (1:400; cat. no. BA-1000; Vector Laboratories, Inc., Burlingame, CA, USA) and streptavidin peroxidase complex (Vector Laboratories, Inc., Burlingame, CA, USA). Immunostaining was visualized with diaminobenzidine and the specimens were mounted using Polymount.

To demonstrate antigen colocalization, the same G-CSFR antibody was used simultaneously with one of the following monoclonal antibodies: anti-neuronal nuclei (NeuN; dilution, 1:100; cat. no. MAB377; EMD Millipore, Billerica, MA, USA), anti-phospho (p)-STAT3 (Tyr705; 1:100; cat. no. 9131; Cell Signaling Technology, Inc., Danvers, MA, USA) and anti-TRPV1 (1:100; cat. no. MAB5568; EMD Millipore). Sections were immunoreacted for G-CSF as described above and then, a $\mathrm{Cy}^{3}$-conjugated goat anti-rabbit secondary antibody was used (1:500; cat. no. PA-43004, Amersham; GE Healthcare Life Sciences, Chalfont, UK). After washing, sections were incubated for NeuN, p-STAT3, TRPV1 and then a $\mathrm{Cy}^{2}$-conjugated anti-mouse secondary antibody (1:200; cat. 
no. PA-42002, Amersham, GE Healthcare Life Sciences), and counterstained with DAPI. Double-stained sections were analysed with ImageJ software version 1.6.0 (National institutes of Health, Bethesda, USA) and an Axiophot microscope (Carl Zeiss AG, Oberkochen, Germany).

Reverse transcription-quantitative polymerase chain reaction $(R T-q P C R)$. Total RNA was isolated from the ipsilateral and control dorsal horn using TRIzol ${ }^{\circledR}$ Reagent (Thermo Fisher Scientific, Inc., Waltham, MA, USA). cDNA was synthesized from $1 \mu \mathrm{g}$ total RNA using the QuantiTect Reverse Transcription kit (Qiagen GmbH, Hilden, Germany), according to the manufacturer's protocol. cDNA was amplified by qPCR using the following primers: Forward, 5'-ATG ACT CCA CTC ACG GCA AAT TC-3' and reverse, 5'-TGG GGT CTC GCT CCT GGA AGA TG for G-CSFR; forward, 5'-AGTTCAACGGCACAGTCAAG-3' and reverse, 5'-TACTCAGCACCAGCATCACC-3' for GAPDH, targeting G-CSFR (Rn-Csf3r-1-SG, cat. no. QT01592199; Qiagen) and GAPDH. The qPCR amplification was performed using cDNA and Power SYBR Green PCR Master mix (Applied Biosystems; Thermo Fisher Scientific, Inc.). The qPCR conditions were as follows: $10 \mathrm{~min}$ at $95^{\circ} \mathrm{C}$ and 40 cycles of $15 \mathrm{sec}$ at $95^{\circ} \mathrm{C}$ and $1 \mathrm{~min}$ at $60^{\circ} \mathrm{C}$. Relative fold changes in G-CSF gene expression were calculated via the $2^{-\Delta \Delta \mathrm{Cq}}$ method (14) using GAPDH as the reference control for normalization. All experiments were performed 3 times.

Cell culture. SH-SY5Y cells (cat. no. KCLB22266; Korean Cell Line Bank, Seoul, Korea) were routinely cultured in 100-mm dishes at $37^{\circ} \mathrm{C}$ in $5 \% \mathrm{CO}_{2}$ with Dulbecco's modified Eagles medium with GlutaMAX ${ }^{\mathrm{TM}}$ (cat. no. 10566-016; Gibco, Thermo Fisher Scientific Inc.) and $4.5 \mathrm{~g} / 1$ glucose, supplemented with $10 \%$ heat-inactivated fetal bovine serum (cat. no. 26140-079, Gibco, Thermo Fisher Scientific Inc.), $1 \%$ non-essential amino acids, $100 \mathrm{U} / \mathrm{ml}$ penicillin and $100 \mu \mathrm{g} / \mathrm{ml}$ streptomycin. Cells were plated at $5 \times 10^{5} / \mathrm{ml}$ and on the following day, cells were treated with $50 \mu \mathrm{g} / \mathrm{ml} \mathrm{G-CSF}$ (cat. no. \#62005; Calbiochem; Merck KGaA, Darmstadt, Germany).

Immunoblot analysis. Following G-CSF treatment for 0, 1, 3, 6 and 24 h, SH-SY5Y cells were harvested ( $1-5 \times 10^{8}$ cells) and homogenized in lysis buffer $(50 \mathrm{mM}$ Tris- $\mathrm{Cl}, 150 \mathrm{mM} \mathrm{NaCl}$, $0.02 \%$ sodium azide, $100 \mu \mathrm{g} / \mathrm{ml}$ PMSF, $1 \mu \mathrm{g} / \mathrm{ml}$ aprotinin, $1 \%$ Triton $\mathrm{X}-100)$ at $4^{\circ} \mathrm{C}$ for $15 \mathrm{~min}$. Following centrifugation $\left(15,000 \mathrm{x} \mathrm{g}, 4^{\circ} \mathrm{C}\right.$, protein concentrations were determined in the supernatants using a Bicinchoninic acid assay kit (Pierce; Thermo Fisher Scientific, Inc.), according to the manufacturer's protocol. Aliquots containing $25 \mu \mathrm{g}$ protein were resolved by $10 \%$ SDS-PAGE and transferred onto polyvinylidene difluoride membranes. For immunoblotting, membranes were incubated in 5\% skim milk in TBS with $0.3 \%$ Triton X-100 for $1 \mathrm{~h}$ at room temperature to block non-specific binding and then probed with the following primary antibodies at $4^{\circ} \mathrm{C}$ overnight: Rabbit anti-G-CSF3 receptor (G-CSFR) polyclonal antibody (dilution, 1:500; cat. no. \#bs-2574R; BIOSS), anti- $\beta$-actin (dilution, 1:5,000; cat. no. \#A5316; Sigma; Merck KGaA), STAT3 (124H6) mouse monoclonal antibody (dilution, 1:1,000; cat. no. \#9139; Cell Signaling Technology, Inc.), anti-p-STAT3 (Tyr705; M9C6) mouse (dilution, 1:200; cat. no. \#4113; Cell
Signaling, Inc.) and anti-capsaicin receptor (the TRPV1 antibody; dilution, 1:1,000; cat. no. MAB5568; EMD Millipore). Membranes were washed three times for $15 \mathrm{~min}$ in TBS with $0.3 \%$ Triton $\mathrm{X}-100$ and incubated for $2 \mathrm{~h}$ at room temperature with horse radish peroxidase-labeled secondary antibodies (1:3,000, cat. no. LF-SA8001, anti-mouse IgG; 1:5,000, cat. no. LF_SA8002, anti-rabbit IgG; Abfrontier, Seoul, Republic of Korea) diluted in 5\% skim milk. Following three additional washes, immunolabeled proteins were detected by chemiluminescence using the Supersignal ECL kit (Pierce; Thermo Fisher Scientific, Inc.), according to the manufacturer's protocol. Western blotting was performed in triplicate for each G-CSF treatment time point. All western blots were subjected to different exposure times, from $10 \mathrm{sec}$ to $5 \mathrm{~min}$, and those with the best exposure were selected for data presentation. The densities of the bands were quantified by ImageJ (64-bit) software version 1.6.0 (National Institutes of Health, Bethesda, MD, USA.

Quantification and statistical analysis. Results from the behavioral study, RT-qPCR and immunoblotting were statistically analyzed using a one-way or two-way analysis of variance (ANOVA). When significant differences were identified by ANOVA, pair-wise comparisons of the means were assessed by the post-hoc Tukey's test. The statistical software package SigmaStat version 3.5 (Systat Software, Inc., San Jose, CA, USA) was used to perform all statistical analyses. Data are presented as the mean \pm standard error mean. $\mathrm{P}<0.05$ was considered to indicate a statistically significant difference.

\section{Results}

SNL induces hypersensitivity in the ipsilateral hind paw. To determine mechanical hypersensitivity following SNL, rat PWTs were measured using the Von Frey hair method. PWT in response to Von Frey hair stimulation decreased in the ipsilateral side following SNL surgery, while sham-operated rats did not exhibit any signs of hypersensitivity (Fig. 1A). SNL induced mechanical hypersensitivity and resulted in a significant decrease in PWTs on the ipsilateral side from day 7 post-surgery when compared with the sham ipsilateral side, which was maintained for up to 14 days. However, this result was not observed on the contralateral side $(\mathrm{P}<0.001$; Fig. 1A). The print area of the ipsilateral hind paws, analyzed by the CatWalk method, was also significantly reduced in ipsilateral SNL-operated rats $(\mathrm{P}<0.05$ and $\mathrm{P}<0.001$; Fig. 1B) compared to ipsilateral sham-operated rats. No significant changes in either the PWTs or print areas were detected in the sham-operated group (ipsilateral vs. contralateral side) or on the contralateral side (SNL vs. sham). The results demonstrate that SNL may induce hypersensitivity and mechanical allodynia in rats.

G-CSFR expression increases in the spinal dorsal horns of rats following $S N L$. The protein and $\mathrm{mRNA}$ expression levels of G-CSFR in the spinal dorsal horn following SNL were assessed by immunoblotting, qPCR and immunohistochemistry using one-quarter of the lumbar spinal cord (Fig. 2). SNL surgery induced a significant upregulation of G-CSFR protein and mRNA expression in the ipsilateral spinal dorsal 

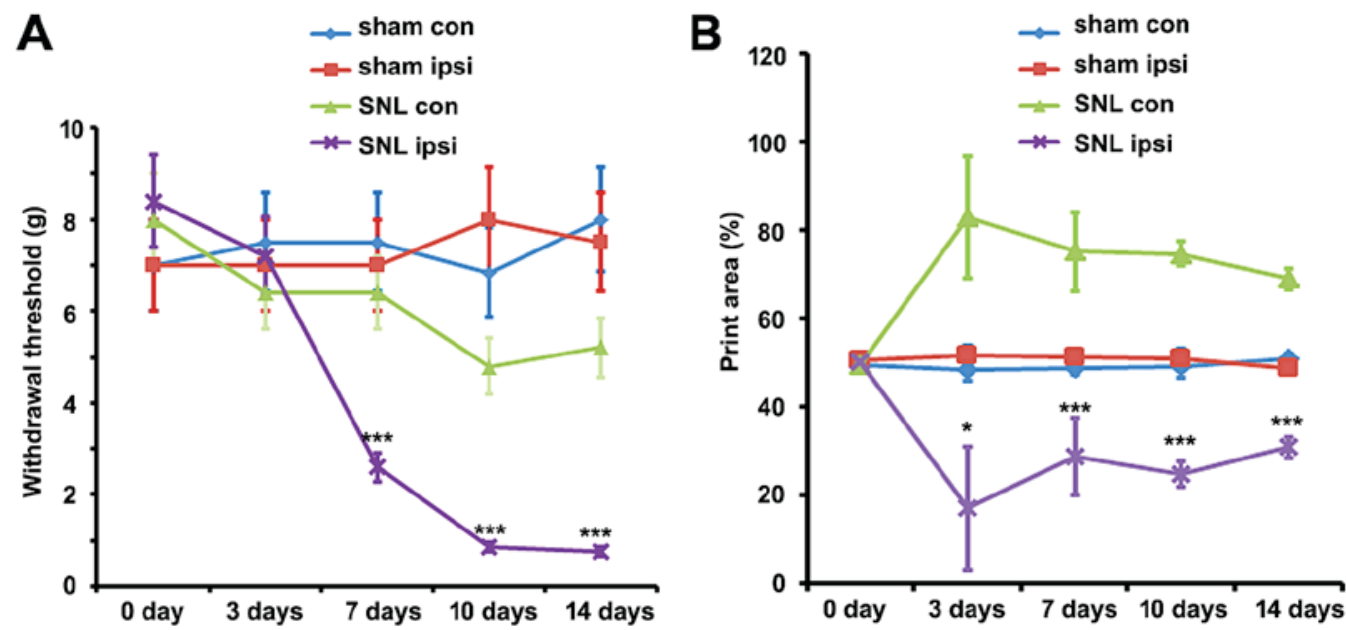

Figure 1. SNL induces hypersensitivity in the rat ipsilateral hind paw. (A) Paw withdrawal threshold in response to Von Frey hair stimulation decreased ipsilaterally following surgery. A severe and persistent mechanical allodynia developed and was maintained over time following SNL. Sham-operated rats did not exhibit any signs of hypersensitivity. Data are expressed as the mean \pm standard error mean of $50 \%$ of the pain threshold and were normalized to the baseline of each animals (B) Analysis of the print area using the CatWalk system indicated that there were significant differences between the ipsilateral and contralateral paws following SNL. The print area of the ipsilateral hind paws was significantly reduced in the SNL model compared with sham group. Data are presented as the mean \pm standard error mean ( $\mathrm{n}=7 /$ group). ${ }^{*} \mathrm{P}<0.05$ and ${ }^{* * * *} \mathrm{P}<0.001$ vs. the corresponding ipsilateral side. SNL, spinal nerve ligation; Con, contralateral hind paw; Ipsi, ipsilateral hind paw; Days, the number of days following SNL or sham surgery.

A

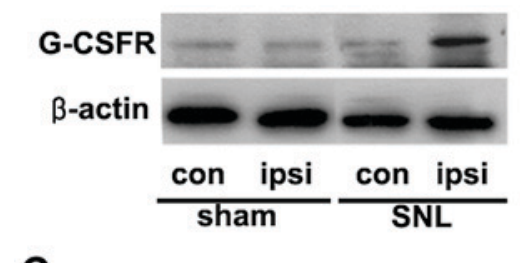

C
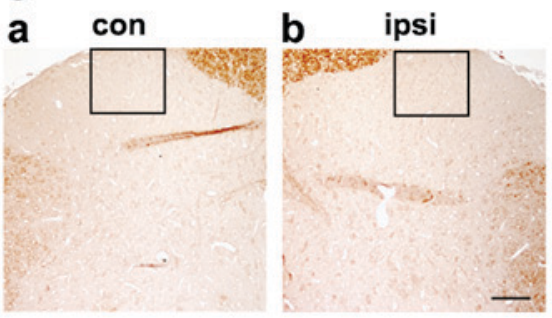

B
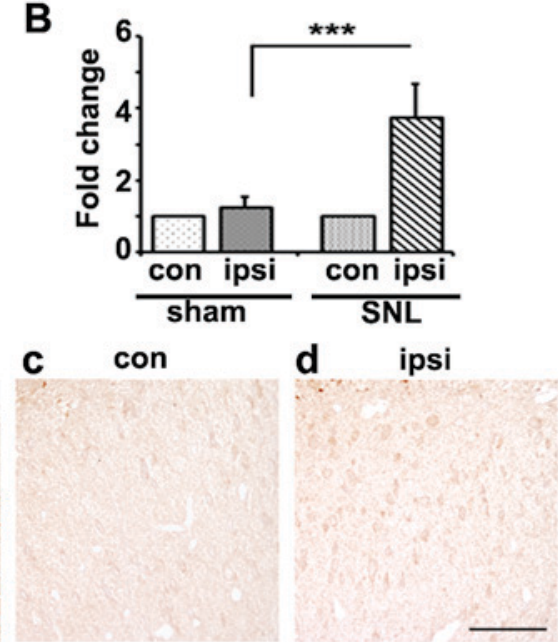

Figure 2. G-CSF expression increased in the spinal cord dorsal horn of rats following SNL. (A) The level of G-CSFR was measured in the lumbar dorsal horn by immunoblotting. Western blotting for G-CSF following SNL or sham surgery demonstrated that G-CSF expression was markedly increased in the ipsilateral dorsal horn when compared with the contralateral horn 14 days following surgery. (B) Reverse transcription-quantitative polymerase chain reaction analysis revealed that G-CSF mRNA expression significantly increased in the ipsilateral dorsal horn when compared with the contralateral horn following surgery. Data are presented as the mean \pm standard error mean ( $\mathrm{n}=7$ rats/group). ${ }^{* * * *} \mathrm{P}<0.001 \mathrm{vs}$. the corresponding ipsilateral side (C) When compared to the (Ca) contralateral spinal dorsal horn, immunoreactive staining revealed that there was L5 SNL induced G-CSFR upregulation in the (Cb) ipsilateral lumbar spinal dorsal horn; G-CSFR staining was weakly observed in the contralateral dorsal horn (scale bar=40 mm). The images within the black rectangles are also shown at a higher magnification. G-CSFR immunoreactive staining in the (Cc) contralateral and (Cd) ipsilateral lumbar spinal dorsal horn, respectively (scale bars=20 mm). G-CSF, granulocyte-colony stimulating factor; G-CSFR, granulocyte-colony stimulating factor receptor; SNL, spinal nerve ligation; Con, contralateral dorsal horn; Ipsi, ipsilateral dorsal horn.

horn however, not in the contralateral horn (Fig. 2A and B) compared to sham group. The expression of G-CSFR was examined in the spinal cord 14 days after L5 SNL by immunohistochemical analysis: SNL induced upregulation of G-CSFR in the ipsilateral spinal cord, particularly in the superficial lumbar dorsal horn, while only a few G-CSFR immunoreactive (IR) cells were detected in the contralateral spinal dorsal horn (Fig. 2C). There were no significant differences in G-CSFR expression in the spinal dorsal horn between the Sham and Naïve groups (data not shown). These results indicate that G-CSFR may serve an important role in the nociceptive sensory pathway of SNL-induced neuropathic pain.

Expression and localization of G-CSFR in the spinal dorsal horn following $S N L$. Double immunofluorescent staining was performed with antibodies against G-CSFR and the neuron marker NeuN to detect the cellular localization of G-CSFR 


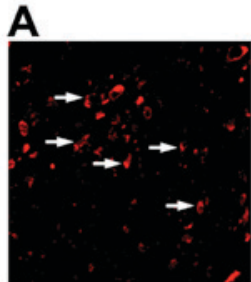

G-CSFR

B

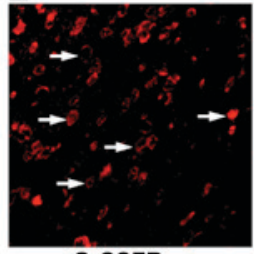

G-CSFR

C

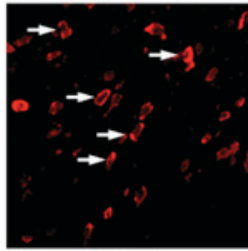

G-CSFR

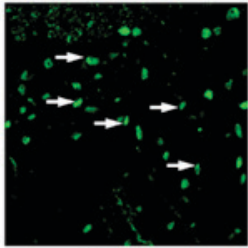

NeuN

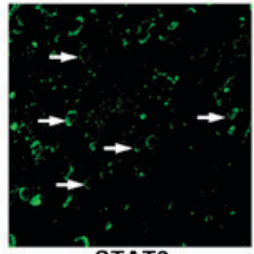

p-STAT3

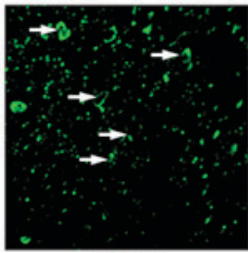

TRPV1

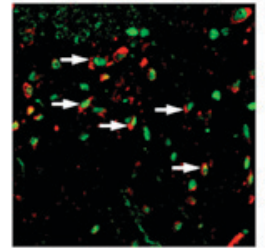

G-CSFR/NeuN

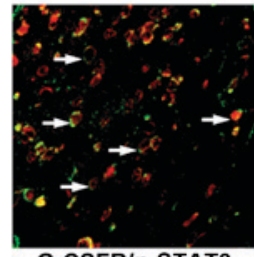

G-CSFR/p-STAT3

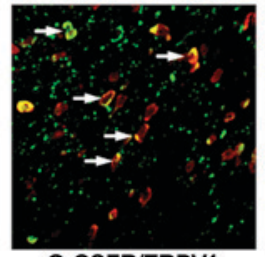

G-CSFR/TRPV1

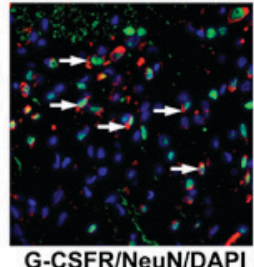

G-CSFR/NeuN/DAPI
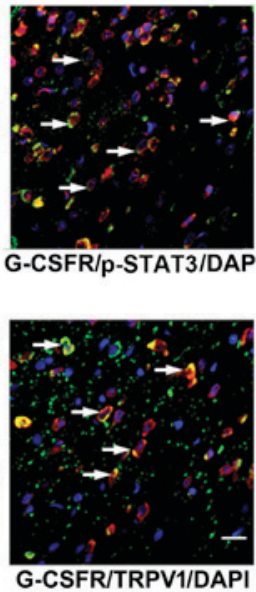

Figure 3. Double immunofluorescent staining of G-CSF (red) with NeuN, p-STAT3 and TRPV1 (green) in rats 14 days following SNL. (A) Double immunofluorescence staining for G-CSFR-positive cells in the ipsilateral spinal dorsal horn following SNL. Immunoreactive G-CSFR cells colocalized with NeuN, a neuronal marker of the superficial dorsal horn (indicated by white arrows). Double immunofluorescence demonstrated that G-CSFR colocalized with (B) p-STAT3 and (C) TRPV1 in the spinal dorsal horn following SNL treatment (indicated by arrows). Scale bar=20 $\mu \mathrm{m}$. G-CSF, granulocyte-colony stimulating factor; G-CSFR, granulocyte-colony stimulating factor receptor; SNL, spinal nerve ligation; NeuN, neuronal nuclei; p-STAT3, phospho-signal transducer and activator of transcription 3; TRPV1, transient receptor potential cation channel subfamily V 1.

expression. G-CSFR IR cells were colocalized completely with $\mathrm{NeuN}$ in the superficial dorsal horn (Fig. 3A). The results of these double-labeling experiments indicated that G-CSFR was expressed in neuronal cells of spinal dorsal horn. In addition, the present study examined whether G-CSFR colocalized with p-STAT3 and TRPV1, as they are important mediators of the pain response. This analysis revealed that G-CSFR fully colocalized with p-STAT3 and TRPV1 expression in the ipsilateral spinal dorsal horn following SNL (Fig. 3B and C). These results support the notion that G-CSFR has an important role in the nociceptive sensory pathway of SNL-induced neuropathic pain via the p-STAT3/TRPV1 signaling pathway.

G-CSF increases G-CSFR, $p$-STAT3 and TRPV1 expression in neuronal cells. As G-CSFR, p-STAT3 and TRPV1 were specifically expressed in neurons in the spinal dorsal horn, the action of G-CSFR on p-STAT3 and TRPV1 was monitored in neurons following G-CSF treatment. As shown in Fig. 4, treatment of SH-SY5Y neurons with $50 \mu \mathrm{g} / \mathrm{ml} \mathrm{G-CSF}$ treatment resulted in significant G-CSFR expression from 1-6 h, compared with $0 \mathrm{~h}$ (Fig. 4A and B). In addition, STAT3 phosphorylation was also significantly increased in neurons from 1-24 h compared with $0 \mathrm{~h}$ (Fig. 4A and C), as was TRPV1 expression (Fig. 4A and D). However, their peak time of expression was at $3 \mathrm{~h}$ whereas G-CSFR expression peaked at $1 \mathrm{~h}$. These results indicated that G-CSF-induced G-CSFR signaling increases the expression of TRPV1 via STAT3 phosphorylation.

\section{Discussion}

In the present study, the results demonstrated that G-CSFR was upregulated in the spinal dorsal horn following SNL-induced neuropathic pain, and that G-CSF/G-CSFR signaling induced the activation of STAT3 and TRPV1 in vitro. Collectively, these results suggest that high levels of G-CSF may mediate the G-CSF/G-CSFR signaling pathway to affect sensory neurons and contribute to the hypersensitivity characteristic of neuropathic pain.

CRPS is a chronic, progressive and devastating pain syndrome characterized by spontaneous pain, hyperalgesia, allodynia, altered skin temperature and motor dysfunction. CRPS type I may develop following trauma with minor or absent nerve damage. CRPS type II is more likely to develop following trauma with clearly detectable peripheral nerve lesions (15). Previously, we have reported that G-CSFR expression levels are significantly different between CRPS patients and controls (10). G-CSFR expression was $2.3 \pm 0.48$-fold higher in the CRPS group when compared with the control group. Gene expression levels in the CRPS type I and CRPS type II subgroups were analysed by comparing the fold change values of CRPS type I or CRPS type II patients with controls. The expression level of G-CSFR was not statistically different between the CRPS type I and control groups however, it was significantly increased (3.6 \pm 0.89 -fold) in patients with CRPS type II when compared with controls (10). To verify the function of upregulated G-CSFR expression in CRPS patients, the present study used an SNL model. This SNL model has been 

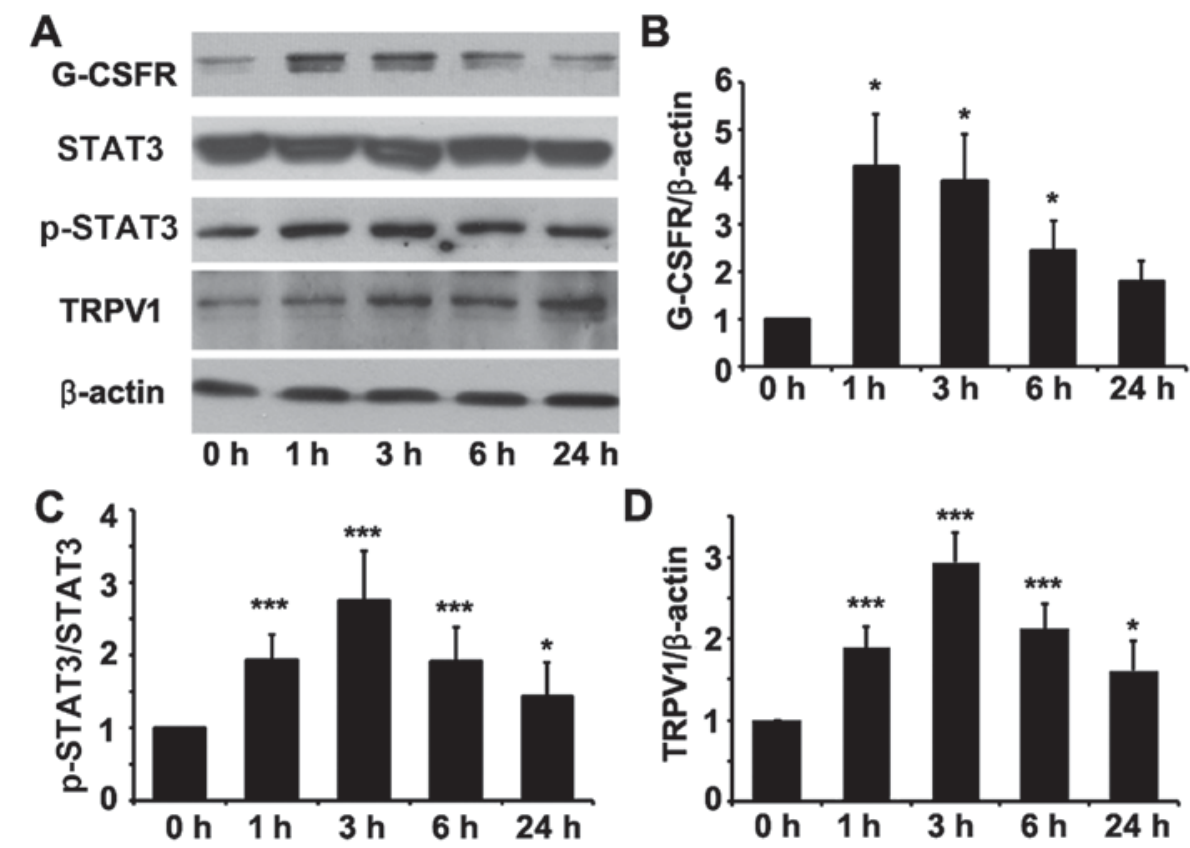

Figure 4. G-CSF treatment increased G-CSFR expression, phosphorylation of STAT3 and TRPV1 expression in neurons. (A) Western blotting time course analysis of G-CSF, STAT3, p-STAT3, TRPV1 and b-actin following treatment with $50 \mu \mathrm{g} / \mathrm{ml} \mathrm{G-CSF}$ in the SH-SY5Y neuronal cell line. Relative density was obtained by densitometry of the corresponding immunoblot data. Statistical differences between (B) G-CSFR, (C) p-STAT3 and (D) TRPV1 were determined by comparing values for $\beta$-actin at each lane. Data are expressed as optical densities and represent the mean \pm standard error mean of three independent experiments. ${ }^{*} \mathrm{P}<0.05$ and ${ }^{* * * *} \mathrm{P}<0.001$ vs. $0 \mathrm{~h}$. G-CSF, granulocyte-colony stimulating factor; G-CSFR, granulocyte-colony stimulating factor receptor; -STAT3, phospho-signal transducer and activator of transcription 3; TRPV1, transient receptor potential cation channel subfamily V 1.

widely used in a number of investigations on neuropathic pain mechanisms and in screening analyses for the development of novel analgesic drugs (16). This model consists of tight ligation of one (lumbar 5) or two (lumbar 5 and lumbar 6) segmental spinal nerves in rats. Therefore, this SNL model is suitable for: 1) the study of central sensitization of pain in patients with CRPS type II, as they have detectable peripheral nerve lesions, and 2) demonstrating long-lasting behavioral signs of mechanical allodynia, heat hyperalgesia, cold allodynia and ongoing pain.

Out of the upregulated gene candidates in patients with CRPS, the present study chose to focus on the role of G-CSFR in central sensitization as its function in pain remains controversial. Huang and Tsai (17) reported that G-CSF treatment induced anti-inflammatory effects in a rat model of anterior ischemic optic neuropathy. However, it has also been reported that treatment with recombinant mouse ( $\mathrm{rm}$ ) G-CSF increased the levels of a number of cytokines and opioid peptides, and exacerbated neuropathic pain in a partial SNL (PSNL) murine model (18). Liou et al (18) revealed that subcutaneous injection of rmG-CSF exacerbated thermal hyperalgesia and tactile allodynia induced by nerve injury, resulting in increased inflammatory cell infiltration and proinflammatory cytokine production in injured nerves following PSNL. However, they did not demonstrate the mechanisms by which this may occur and, in particular, did not address the contribution of G-CSFR to pain hypersensitivity. To the best of our knowledge, the present study has demonstrated for the first time that G-CSFR expression increases in the spinal dorsal horn of rats following SNL surgery. In addition, G-CSFR completely colocalized with p-STAT and TRPV1 in the neurons of the spinal dorsal horn. Furthermore, G-CSF treatment led to the increased expression of G-CSFR and TRPV1, and increased phosphorylation of STAT3. As TRPV1 is a nonselective cation channel expressed in subtypes of nociceptive neurons with known effects on inflammatory and neuropathic pain (19), the results suggest that following peripheral nerve injury, neuronal G-CSFR may be involved in the induction and maintenance of neuropathic pain through the STAT3/TRPV1 signaling pathway.

The role of G-CSFR in central spinal cord sensitization in the SNL-induced neuropathic pain model was also evaluated. Central sensitization refers to the process by which a state of hyperexcitability is established in the central nervous system, leading to enhanced processing of nociceptive pain messages. Although a number of mechanisms have been implicated in central sensitization, one key mechanism of neuropathic pain is neuroinflammation $(20,21)$. Proinflammatory cytokines, including IL-1B, IL-6 and tumor necrosis factor- $\alpha$, usually induce or facilitate neuropathic pain. By contrast, blockade of proinflammatory cytokines and/or administration of anti-inflammatory cytokines, including IL-10, IL-2 and IL-4, reduced neuropathic pain in animal models (22). In addition, inflammatory mediators including ATP, trypsin, bradykinin, prostaglandin E2 and nerve growth factor can induce a dynamic reduction in the threshold of TRPV1 activation $(23,24)$. Previous studies have indicated that at the spinal cord level, TRPV1 regulates the release of neurotransmitters, such as substance $\mathrm{P}$ and calcitonin gene-related peptide $(25,26)$. It has been established that TRPV1 modulation is relevant to tissue injury-evoked pain hypersensitivity, particularly in the setting of inflammation. These conditions can include sunburn, infection, rheumatoid arthritis or osteoarthritis, and inflammatory bowel disease. Another notable example includes pain from bone cancer, in 
which tumor growth and bone destruction are accompanied by extremely robust tissue acidosis, as well as production of cytokines, neurotrophins and prostaglandins (27). The present study indicates that G-CSF treatment may be detrimental to patients with neuropathic pain as G-CSFR signaling and TRPV1 modulation are relevant to tissue injury-evoked pain hypersensitivity. More detailed studies on G-CSFR antagonists and G-CSF absorption are required to assess their potential as therapeutic treatments for neuropathic pain.

\section{Acknowledgements}

The present study was supported by the Basic Science Research Program through the National Research Foundation of Korea funded by the Ministry of Science, ICT and Future Planning (grant nos.2013R1A1A1A05006966 and 2014R1A1A1038222) and in part by Chungnam National University Hospital Research Fund, 2014.

\section{References}

1. Nagata S, Tsuchiya M, Asano S, Kaziro Y, Yamazaki T, Yamamoto O, Hirata Y, Kubota N, Oheda M, Nomura H, et al: Molecular cloning and expression of cDNA for human granulocyte colony-stimulating factor. Nature 319: 415-418, 1986.

2. Fukunaga R, Ishizaka-Ikeda E, Seto Y and Nagata S: Expression cloning of a receptor for murine granulocyte colony-stimulating factor. Cell 61: 341-350, 1990.

3. Mine S, Koshiba T, Honjo E, Okamoto T, Tamada T, Maeda Y, Matsukura Y,Horie A,Ishibashi M,Sato M, et al: Thermodynamic analysis of the activation mechanism of the GCSF receptor induced by ligand binding. Biochemistry 43: 2458-2464, 2004.

4. Marino VJ and Roguin LP: The granulocyte colony stimulating factor (G-CSF) activates Jak/STAT and MAPK pathways in a trophoblastic cell line. J Cell Biochem 103: 1512-1523, 2008.

5. Shimoda K, Feng J, Murakami H, Nagata S, Watling D, Rogers NC, Stark GR, Kerr IM and Ihle JN: Jak1 plays an essential role for receptor phosphorylation and Stat activation in response to granulocyte colony-stimulating factor. Blood 90: 597-604, 1997.

6. Würfel W: Treatment with granulocyte colony-stimulating factor in patients with repetitive implantation failures and/or recurrent spontaneous abortions. J Reprod Immunol 108: 123-135, 2015.

7. Kirsch F, Krüger C and Schneider A: The receptor for granulocyte-colony stimulating factor (G-CSF) is expressed in radial glia during development of the nervous system. BMC Dev Biol 8: 32,2008 .

8. Ridwan S, Bauer H, Frauenknecht K, Hefti K, von Pein H and Sommer CJ: Distribution of the hematopoietic growth factor G-CSF and its receptor in the adult human brain with specific reference to Alzheimer's disease. J Anat 224: 377-391, 2014.

9. Schneider A, Krüger C, Steigleder T, Weber D, Pitzer C, Laage R, Aronowski J, Maurer MH, Gassler N, Mier W, et al: The hematopoietic factor G-CSF is a neuronal ligand that counteracts programmed cell death and drives neurogenesis. J Clin Invest 115: 2083-2098, 2005.

10. Jin EH, Zhang E, Ko Y, Sim WS, Moon DE, Yoon KJ, Hong JH and Lee WH: Genome-wide expression profiling of complex regional pain syndrome. PLoS One 8: e79435, 2013.
11. Schweizerhof M, Stösser S, Kurejova M, Njoo C, Gangadharan V, Agarwal N, Schmelz M, Bali KK, Michalski CW, Brugger S, et al: Hematopoietic colony-stimulating factors mediate tumor-nerve interactions and bone cancer pain. Nat Med 15: 802-807, 2009.

12. Baron R: Neuropathic pain: A clinical perspective. Handb Exp Pharmacol: 3-30, 2009.

13. Kim SH and Chung JM: An experimental model for peripheral neuropathy produced by segmental spinal nerve ligation in the rat. Pain 50: 355-363, 1992

14. Livak KJ and Schmittgen TD: Analysis of relative gene expression data using real-time quantitative PCR and the 2(-Delta Delta C(T)) Method. Methods 25: 402-408, 2001.

15. Jänig $\mathrm{W}$ and Baron R: Complex regional pain syndrome is a disease of the central nervous system. Clin Auton Res 12: $150-164,2002$

16. Chung JM, Kim HK and Chung K: Segmental spinal nerve ligation model of neuropathic pain. Methods Mol Med 99: 35-45, 2004.

17. Huang SP and Tsai RK: Efficacy of granulocyte-colony stimulating factor treatment in a rat model of anterior ischemic optic neuropathy. Neural Regen Res 9: 1502-1505, 2014.

18. Liou JT, Lui PW, Liu FC, Lai YS and Day YJ: Exogenous granulocyte colony-stimulating factor exacerbate pain-related behaviors after peripheral nerve injury. J Neuroimmunol 232: 83-93, 2011

19. Lima CK, Silva RM, Lacerda RB, Santos BL, Silva RV, Amaral LS, Quintas LE, Fraga CA, Barreiro EJ, Guimaraes MZ and Miranda AL: LASSBio-1135: A dual TRPV1 antagonist and anti-TNF-alpha compound orally effective in models of inflammatory and neuropathic pain. PLoS One 9: e99510, 2014.

20. Chen NF, Huang SY, Lu CH, Chen CL, Feng CW, Chen CH, Hung HC, Lin YY, Sung PJ, Sung CS, et al: Flexibilide obtained from cultured soft coral has anti-neuroinflammatory and analgesic effects through the upregulation of spinal transforming growth factor- $\beta 1$ in neuropathic rats. Mar Drugs 12: 3792-3817, 2014.

21. Walters ET: Neuroinflammatory contributions to pain after SCI: Roles for central glial mechanisms and nociceptor-mediated host defense. Exp Neurol 258: 48-61, 2014.

22. Shao Q, Li Y, Wang Q and Zhao J: IL-10 and IL-1 $\beta$ mediate neuropathic-pain like behavior in the ventrolateral orbital cortex. Neurochem Res 40: 733-739, 2015.

23. Ma W and Quirion R: Inflammatory mediators modulating the transient receptor potential vanilloid 1 receptor: Therapeutic targets to treat inflammatory and neuropathic pain. Expert Opin Ther Targets 11: 307-320, 2007.

24. Szallasi A, Cortright DN, Blum CA and Eid SR: The vanilloid receptor TRPV1: 10 years from channel cloning to antagonist proof-of-concept. Nat Rev Drug Discov 6: 357-372, 2007.

25. Kanai Y, Nakazato E, Fujiuchi A, Hara T and Imai A: Involvement of an increased spinal TRPV1 sensitization through its up-regulation in mechanical allodynia of CCI rats. Neuropharmacology 49: 977-984, 2005.

26. Puttfarcken PS, Han P, Joshi SK, Neelands TR, Gauvin DM, Baker SJ, Lewis LG, Bianchi BR, Mikusa JP, Koenig JR, et al: A-995662 [(R)-8-(4-methyl-5-(4-(trifluoromethyl)phenyl) oxazol-2-ylamino)-1,2,3,4-tetrahydronaphthalen-2-ol], a novel, selective TRPV1 receptor antagonist, reduces spinal release of glutamate and CGRP in a rat knee joint pain model. Pain 150: 319-326, 2010.

27. Honore P, Chandran P, Hernandez G, Gauvin DM, Mikusa JP, Zhong C, Joshi SK, Ghilardi JR, Sevcik MA, Fryer RM, et al: Repeated dosing of ABT-102, a potent and selective TRPV1 antagonist, enhances TRPV1-mediated analgesic activity in rodents, but attenuates antagonist-induced hyperthermia. Pain 142: 27-35, 2009. 